\title{
An autochthonous case of cystic echinococcosis in Finland, 2015
}

S Hämäläinen ${ }^{1}$, A Kantele ${ }^{23}$, M Arvonen ${ }^{4}$, T Hakala 5 , J Karhukorpi ${ }^{6}$, J Heikkinen ${ }^{7}$, E Berg ${ }^{8}$, K Vanamo ${ }^{9}$, E Tyrväinen ${ }^{10}$, T

Heiskanen-Kosma ${ }^{4}$, A Oksanen ${ }^{11}$, A Lavikainen ${ }^{12}$

1. Department of Medicine, Kuopio University Hospital, Kuopio, Finland

2. Inflammation Center, Clinic of Infectious Diseases, University of Helsinki and Helsinki University Hospital, Helsinki, Finland

3. Unit of Infectious Diseases, Karolinska Institutet, Solna, Sweden

4. Department of Pediatrics, Kuopio University Hospital, Kuopio, Finland

5. Department of Surgery, North Karelia Central Hospital, Joensuu, Finland

6. Eastern Finland Laboratory Centre Joint Authority Enterprise (ISLAB), Joensuu District Laboratory, Joensuu, Finland

7. Department of Internal Medicine, North Karelia Central Hospital, Joensuu, Finland

8. Heart Center, Kuopio University Hospital, Kuopio, Finland

9. Department of Pediatric Surgery, Kuopio University Hospital, Kuopio, Finland

10. Department of Clinical Radiology, Kuopio University Hospital, Kuopio, Finland

11. Finnish Food Safety Authority Evira/FINPAR, Oulu, Finland

12. Department of Bacteriology and Immunology/Immunobiology Program/FINPAR, Faculty of Medicine, University of Helsinki, Helsinki, Finland

Correspondence: Antti Lavikainen (antti.lavikainen@helsinki.fi)

Hämäläinen S, Kantele A, Arvonen M, Hakala T, Karhukorpi J, Heikkinen J, Berg E, Vanamo K, Tyrväinen E, Heiskanen-Kosma T, Oksanen A, Lavikainen

A. An autochthonous case of cystic echinococcosis in Finland, 2015. Euro Surveill. 2015;20(42):pii=30043. DOI: http://dx.doi.org/10.2807/1560-7917.

ES.2015.20.42.30043

Article submitted on 07 October 2015 / accepted on 20 October 2015 / published on 22 October 2015

We report a case of pulmonary cystic echinococcosis in a child from eastern Finland with no history of travelling abroad. The cyst was surgically removed and the organism molecularly identified as Echinococcus canadensis genotype $\mathrm{G}_{10}$. This parasite is maintained in eastern Finland in a sylvatic life cycle involving wolves and moose; in the present case, the infection was presumably transmitted by hunting dogs.

In Fennoscandia (Finland, Norway and Sweden) and parts of western central Europe, cystic echinococcosis (CE) or hydatidosis is a rare disease seen in immigrants or other people who have resided in endemic countries. Here we present an unexpected autochthonous case of pulmonary CE in a Finnish child.

\section{Case description}

At the end of February 2015, an eight-year-old previously healthy child from eastern Finland had sudden abdominal pain and developed a vigorous generalised urticarial rash without other abnormal findings. The child had never travelled abroad. A week later, the child was admitted to hospital because of fever $\left(38.5^{\circ} \mathrm{C}\right)$ and a persistent cough. Upon admission, the patient was pale and their breath sounds were decreased over the left side of the chest. A chest $X$-ray revealed a large cavity, partially filled with fluid (Figure 1A). The ultrasound showed a considerable avocado-sized hollow $(13.5 \times 9 \mathrm{~cm})$ with multiple lobulation. Consistent with these findings and an elevated C-reactive protein (58 $\mathrm{mg} / \mathrm{L}$; norm: $<3 \mathrm{mg} / \mathrm{L})$, and high normal leukocyte count (12.9 $\times 10^{9} / \mathrm{L}$; norm: 4.5-13.5 $\left.\times 10^{9} / \mathrm{L}\right)$, a lung abscess with parapneumonic empyema was set as the principal diagnosis. Elevation of the serum eosinophil leucocytes (4.7 $\times 10^{9} / \mathrm{L}$; norm: $\left.0.1-0.4 \times 10^{9} / \mathrm{L}\right)$ was also observed.

Despite empirical treatment with intravenous cefuroxime (100 mg/kg/day divided in three doses) and clindamycin (40 mg/kg/day divided in three doses), the fever and cough persisted and the radiological findings did not resolve within a week. Computed tomography (CT) of the thorax on day 5 after admission revealed a pleural effusion and an empyema in the lower lobe of the left lung, and an abscess suspicion in the upper lobe (Figure 1B). A left-sided thoracotomy was performed on day 7 after admission. An empyema was detected and debridement of the pleural cavity and decortication was carried out. The suspected abscess cavity was opened and partially resected. Clinically, it proved to be a cystic structure with connection to small bronchi. This bronchocystic fistula was closed with sutures.

On the first postoperative day, the direct microscopic examination of calcofluor white-stained fragments of cyst wall and cyst content showed plenty of hooks and protoscolices, typical of Echinococcus. Hooks were detected also in Gram (Figure 2) and Ziehl-Neelsen stained samples. After this finding, albendazole treatment (10-15 mg/kg/day divided in two doses) was started. Because of the obvious CE, a re-thoracotomy was performed on the second postoperative day. The cyst was close to the hilar structures and thus the upper lobe of the left lung had to be removed. 


\section{FIGURE 1}

Chest X-ray and computed tomography, autochthonous cystic echinococcosis case in a child, east Finland, March 2015

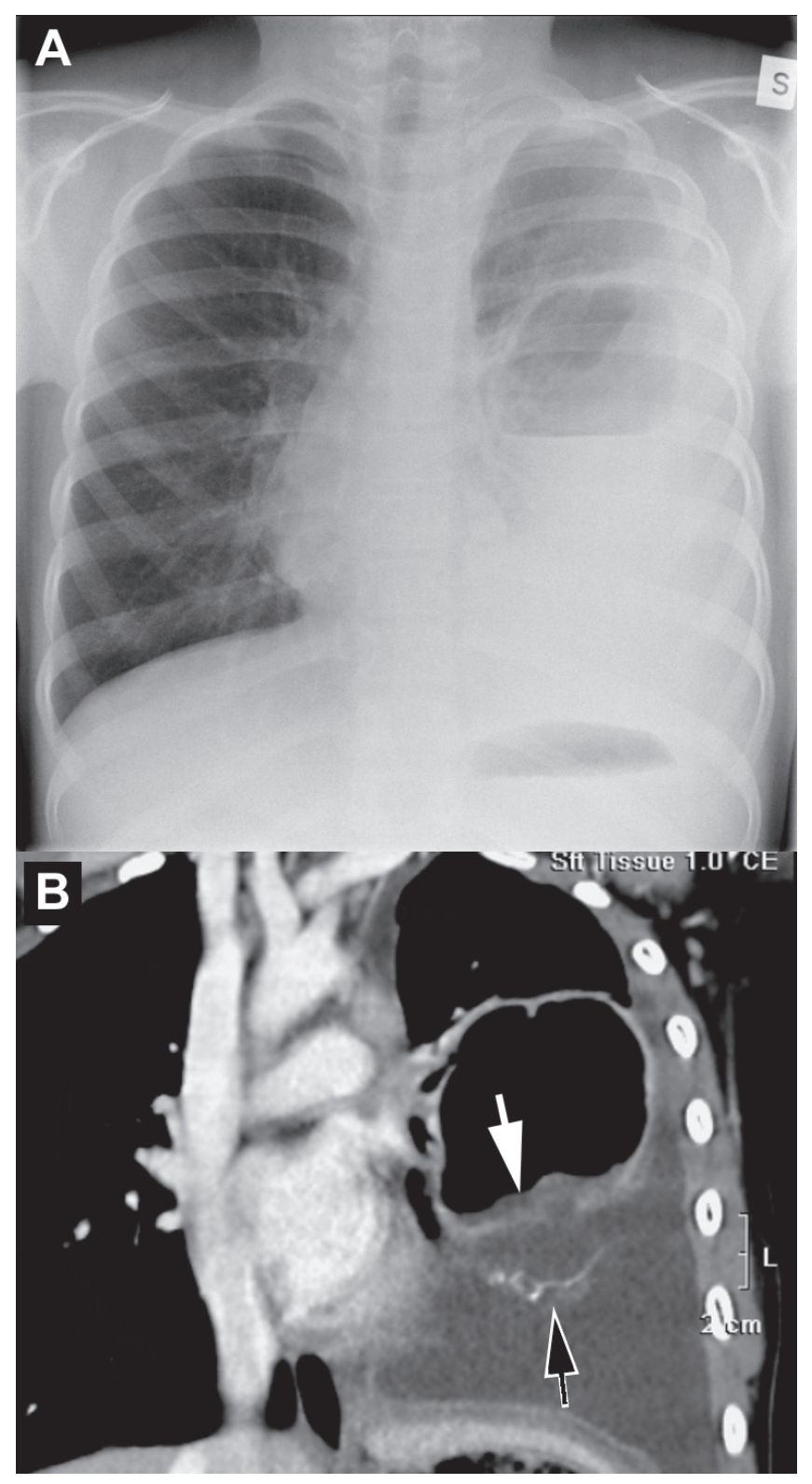

A: Chest X-ray showing pleural effusion and a large cyst with airfluid level in the upper lobe of the left lung. The cyst wall is thick and slightly irregular.

B: Coronal reconstruction image from contrast-enhanced computed tomography showing the cyst and pleural effusion. The cyst wall is enhanced; the collapsed endocyst is indicated by the white arrow and the small calcifications are indicated by the black arrow.

PCR for Echinococcus (partial mitochondrial cytochrome $c$ subunit I gene [1]) was positive in specimens taken during the first thoracotomy from both the cyst and pleural empyema, indicating either spontaneous or intraoperative spillage of the cyst content into the pleural cavity. Sequencing revealed $100 \%$ identity with Echinococcus canadensis genotype $\mathrm{G} 10$ previously isolated in cervids from Finland [2].
Postoperatively, the lung function recovered slowly. A thorax and abdomen CT and an abdominal ultrasound did not reveal signs of hydatid cysts in the liver or other parts of the body. The findings in brain magnetic resonance imaging (MRI) were also normal. A serum sample taken six weeks after the first operation was strongly positive for IgG antibodies against $E$. granulosus by ELISA and IHA (Swiss Tropical and Public Health Institute, Basel, Switzerland). The tests are based on material of $E$. granulosus sensu stricto (s.s.) genotype $\mathrm{G} 1$ of sheep originating in Sardinia (Bruno Gottstein, University of Bern). Albendazole treatment was continued postoperatively for a total of three months.

\section{Investigation of close family members and dogs owned by the family}

The patient's parents and two siblings were examined for Echinococcus infection. Chest X-rays and abdominal ultrasound results proved normal and serological tests were negative. The family lives in the countryside and has three dogs, which are used mostly for fowling. There was no history of feeding dogs with raw cervid viscera. The dogs were regularly dewormed, but not with anthelmintics effective against Echinococcus. Before treatment with praziquantel, faecal samples were collected from the dogs. Mitochondrial DNA was extracted directly from the faecal material [3] because the small size of the samples did not allow isolation of parasite eggs. A fragment of mitochondrial ribosomal DNA of the E. granulosus sensu lato (s.l.) complex [4] was detected by PCR in all the samples, but the short unspecific sequence did not reveal the exact species. Control specimens taken one month after deworming were PCR-negative.

\section{Background}

The most important aetiological agents of human CE are $E$. granulosus S.s. and $E$. canadensis, both of which were formerly included in the species complex of $E$. granulosus s.l [5]. Canids are the definitive hosts of these parasites, and various ungulates serve as intermediate hosts [6].

CE is found worldwide, prevailing in many endemic areas, typically in pastoral communities where people have close contact with dogs [6]. In the northern Fennoscandia, endemic human CE occurred in the reindeer herding area until the second half of the last century [7]. In Finland, the last published autochthonous case was diagnosed in 1963, in Sweden in 1967, and in Norway in 1977 [8]. Human infections derived from a synanthropic cycle involving dogs and reindeer, which broke up due to changes in traditional reindeer husbandry including decline of herding dogs $[7,8]$. A sylvatic wolf-moose cycle still exists in Finland, but it has not been linked to human infections [7].

\section{Discussion}

Since our patient had never travelled abroad, a parasite infection was not initially considered in the differential diagnostics. In endemic areas with high prevalence of 


\section{FIGURE 2}

Gram stain of the cyst wall showing two Echinococcus hooks, autochthonous cystic echinococcosis case in a child, east Finland, March 2015

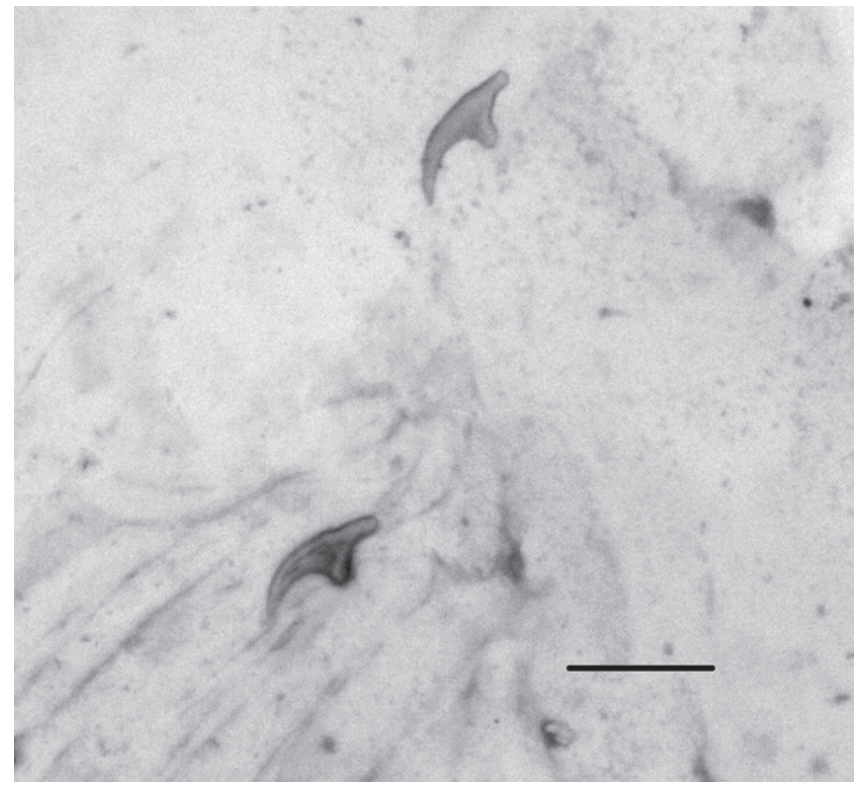

Scale bar $30 \mu \mathrm{m}$.

clinical cases (e.g. in the Middle East or parts of Africa) the eosinophilia, generalised urticaria and pulmonary involvement would be expected to raise a suspicion of CE. The incidental finding of Echinococcus hooks in smears obtained for both mycological and bacterial staining highlights the importance of direct microscopic examination in this case.

The growth rate of hydatid cysts is slow (max. $13 \mathrm{~cm}$ per year, but usually much less) $[9,10]$, depending on location, causative species and probably also patient's age $[9,11,12]$. In the present case, the cyst had presumably been growing for at least one year and eventually constricted the lower lobe, thus predisposing to a secondary pulmonary infection. During this process, a fistula had ruptured and the cyst had been partially drained into the bronchial tree causing a persistent cough. Cyst leakage and exposure to echinococcal antigens probably account for the abdominal pain and urticarial rash.

Based on ultrasound, CT and clinical findings, the present case can be classified as CE4l according to the classification of the World Health Organization Informal Working Group on Echinococcosis (WHO-IWGE) $[9,13]$. In the classification, '4' refers to an inactive stage (with degenerative contents and calcification in the present case) and 'l' to a large size [13]. Most cysts of this type are not fertile [13], but in this case protoscolices were present, indicating fertility.

Surgery still remains the main therapeutic option in pulmonary CE [6]. Here the cyst was not fully removed in the initial operation, since the parasitic aetiology was recognised only after microscopic examination. Due to the large size and difficult location of the cyst, the patient was re-operated and a lobectomy was performed for complete eradication of the parasite, and to avoid relapses and bacterial infections. An optimal length of postoperative albendazole treatment has not been established [9]. However, considering the formation of the fistula and the spilling during the initial operation, a prolonged course of albendazole was considered warranted. Furthermore, follow-up visits including serology are planned every three to six months over the first year and, thereafter, once a year by serology and MRI at least for five years if no clinical symptoms or findings develop.

According to reports from Alaska and Canada, 'sylvatic' (transmitted in wildlife) CE attributed to $E$. canadensis is more benign than classic 'pastoral' CE predominantly caused by $E$. granulosus S.S. in sheepraising countries [11,14-16]. Relative mildness was also typical of endemic CE which occurred in the reindeer herding area of Fennoscandia [8]. The sylvatic form of CE is characterised by pulmonary involvement of relatively small hydatids with thinner laminated membrane (endocyst), and spontaneous cure because of cyst rupture into bronchi $[11,14,15,17]$. Patients are mostly asymptomatic and complications uncommon $[11,14,15]$. Percutaneous aspiration and drainage have been successfully accomplished for therapeutic and diagnostic purposes $[12,15,17]$. Anaphylaxis and secondary seeding have been extremely rare even in spontaneously ruptured cases $[11,14,18]$. Thus, in contrast to the global guidelines [6], in endemic cases in Alaska and Canada surgery is limited to symptomatic, infected or rapidly growing cysts $[11,12,14-17]$. Consistent with the clinical picture of our patient, children are more frequently symptomatic and prone to complications (e.g. bacterial secondary infections) from a cyst rupture, probably because the cysts occupy a large proportion of the lung volume, and the small calibre of bronchi prevents expectoration of parasite remnants [12].

The causative agents of sylvatic CE in the old clinical reports presumably represented the same genotypes (G8 and G10) that occur across the circumpolar north today. So far, G10 has been recorded only in three human cases: the first in southern Mongolia in 2011, the second in north-western Sakha Republic, Russia in 2013, and the most recent one in north-eastern China in 2015 [19-21]. All cases were treated surgically but the clinical course was not presented. The genotype G8 has been reported only once; the patient was operated in southeastern Alaska in 1999 [18,22]. The case, with multiple cysts disseminated in the peritoneal cavity, was atypical as sylvatic CE [18]. It is not known whether these different genotypes of $E$. canadensis correlate with differences in the clinical presentation of sylvatic CE. 
The case presented here most likely originated in the sylvatic cycle via dogs. Given the slow growth of the larval stage and short lifespan of adult parasites, it is unclear whether the cyst in the patient was attributed to the infection detected in the dogs of the patient's family. Although fed only with commercial dog foods, the dogs may have eaten raw viscera, e.g. during hunting, unnoticed by the owner.

Cervid offals are usually discarded at the shooting site. Even though wild scavengers operate rapidly, potentially infected offals are easily available during the moose hunting season for hunting dogs and others roaming freely in the woods. To prevent transmission, raw unfrozen or uncooked cervid viscera are not to be given to dogs, and cestocidic medication is to be regularly administered to hunting dogs before and after the hunting season $[7,23]$. Although sylvatic human $C E$ is rare and sporadic, veterinary authorities should inform hunters and dog owners about these precautions and the potential risk of CE in areas where $E$. canadensis occurs in wildlife. In Europe, this includes Finland, the Baltic countries and Russia [7], and probably also some other countries which belong to the distribution range of wolves.

\section{Conflict of interest}

None declared.

\section{Authors' contributions}

Drafting the manuscript: $\mathrm{SH}, \mathrm{AL}$; commenting on and revising the manuscript: AK, MA, TH, JK, JH, EB, KV, ET, TH-K, AO; attending physicians: $\mathrm{SH}, \mathrm{MA}, \mathrm{TH}, \mathrm{JH}, \mathrm{EB}, \mathrm{KV}$; consulting specialists: AK, TH-K; microbiological diagnosis: JK; genetic identification: $A L$; radiological interpretation: $E T$; veterinary diagnostics: AO.

\section{References}

1. Bowles), BlairD, McManusDP. Genetic variants within the genus Echinococcus identified by mitochondrial DNA sequencing. Mol Biochem Parasitol. 1992;54(2):165-73. DOI: 10.1016/01666851(92)90109-W PMID: 1435857

2. LavikainenA, LehtinenMJ, MeriT, Hirvelä-KoskiV, MeriS. Molecular genetic characterization of the Fennoscandian cervid strain, a new genotypic group (G10) of Echinococcus granulosus.Parasitology. 2003;127(3):207-15. DOI: 10.1017/ So031182003003780 PMID: 12964823

3. IsakssonM, HagströmÅ, Armua-FernandezMT, WahlströmH, ÅgrenEO, MillerA, et al. A semi-automated magnetic capture probe based DNA extraction and real-time PCR method applied in the Swedish surveillance of Echinococcus multilocularis in red fox (Vulpes vulpes) faecal samples. Parasit Vectors. 2014;7(1):583. DOI: 10.1186/S13071-014-0583-6 PMID: 25522844

4. TrachselD, DeplazesP, MathisA. Identification of taeniid eggs in the faeces from carnivores based on multiplex PCR using targets in mitochondrial DNA.Parasitology. 2007:134(06):911 20. DOI: 10.1017/So031182007002235 PMID: 17288631

5. Alvarez RojasCA, RomigT, LightowlersMW. Echinococcus granulosus sensu lato genotypes infecting humans--review of current knowledge.Int J Parasitol. 2014;44(1):9-18. DOI: 10.1016/j.ijpara.2013.08.008 PMID: 24269720

6. Eckert J, Gemmell MA, Meslin F-X, Pawtowski ZS, editors. WHO/ OIE Manual on echinococcosis in humans and animals: a public health problem of global concern. Paris: World Organisation for Animal Health and World Health Organization; 2001.
7. Oksanen A, Lavikainen A. Echinococcus canadensis transmission in the North. Vet Parasitol. Epub 2015 Jul 31 ahead of print.

8. LavikainenA. (Human echinococcosis in Lapland). Ihmisen ekinokokkitauti Suomen, Ruotsin ja Norjan Lapissa. Suomen Eläinlääkärilehti. 2005;110(1):7-13. Finnish.

9. Writing Panel for the WHO-IWGE,BrunettiE, KernP, VuittonDA. Expert consensus for the diagnosis and treatment of cystic and alveolar echinococcosis in humans. Acta Trop. 2010;114(1):1-16. DOI: 10.1016/j.actatropica.2009.11.001 PMID: 19931502

10. RomigT, ZeyhleE, MacphersonCNL, ReesPH, WereJB. Cyst growth and spontaneous cure in hydatid disease.Lancet. 1986;327(8485):861-2. DOI: 10.1016/S0140-6736(86)90974-8 PMID: 2870346

11. WilsonJF, DiddamsAC, RauschRL. Cystic hydatid disease in Alaska. A review of 101 autochthonous cases of Echinococcus granulosus infection.Am Rev Respir Dis. 1968;98(1):1-15.PMID: 5690790

12. LamyAL, CameronBH, LeBlancJG, Gordon CulhamJA, BlairGK, TaylorGP. Giant hydatid lung cysts in the Canadian northwest: outcome of conservative treatment in three children.J Pediatr Surg. 1993;28(9):1140-3. DOI: 10.1016/0022-3468(93)90149-F PMID: 8308679

13. WHO Informal Working Group,Working GroupWHOI. International classification of ultrasound images in cystic echinococcosis for application in clinical and field epidemiological settings.Acta Trop. 2003;85(2):253-61. DOI: 10.1016/So001-706X(02)00223-1 PMID: 12606104

14. MeltzerH, KovacsL, OrfordT, MatasM. Echinococcosis in North American Indians and Eskimos.Can Med Assoc I. 1956;75(2):121-7.PMID: 13343064

15. PinchLW, WilsonJF. Non-surgical management of cystic hydatid disease in Alaska: a review of 30 cases of Echinococcus granulosus infection treated without operation. Ann Surg. 1973;178(1):45-8. DOI: 10.1097/00000658-197307000-00010 PMID: 4736907

16. MooreRD, UrschelJD, FraserRE, NakaiSS, GeeraertAJ. Cystic hydatid lung disease in northwest Canada.Can J Surg. 1994;37(1):20-2.PMID: 8306214

17. FinlayJC, SpeertDP. Sylvatic hydatid disease in children: case reports and review of endemic Echinococcus granulosus infection in Canada and Alaska.Pediatr Infect Dis J. 1992;11(4):322-6. DOI: 10.1097/00006454-199204000-00012 PMID: 1565558

18. CastrodaleLJ, BellerM, WilsonJF, SchantzPM, McManusDP, ZhangL-H, et al. Two atypical cases of cystic echinococcosis (Echinococcus granulosus) in Alaska, 1999. Am J Trop Med Hyg. 2002;66(3):325-7.PMID: 12139230

19. JabbarA, NarankhajidM, NolanMJ, JexAR, CampbellBE, GasserRB. A first insight into the genotypes of Echinococcus granulosus from humans in Mongolia.Mol Cell Probes. 2011;25(1):49-54. DOI: 10.1016/j.mcp.2010.11.001 PMID: 21075201

20. KonyaevSV, YanagidaT, NakaoM, IngovatovaGM, ShoykhetYN, BondarevAY, et al. Genetic diversity of Echinococcus spp. in Russia. Parasitology. 2013;140(13):1637-47. DOI: 10.1017/ So031182013001340 PMID: 23985385

21. YangD, ZhangT, ZengZ, ZhaoW, ZhangW, LiuA. The first report of human-derived $\mathrm{G}_{10}$ genotype of Echinococcus canadensis in China and possible sources and routes of transmission.Parasitol Int. 2015;64(5):330-3. DOI: 10.1016/j. parint.2015.05.001 PMID: 25967082

22. McManusDP, ZhangL, CastrodaleLJ, LeTH, PearsonM, BlairD. Short report: molecular genetic characterization of an unusually severe case of hydatid disease in Alaska caused by the cervid strain of Echinococcus granulosus.Am J Trop Med Hyg. 2002;67(3):296-8.PMID: 12408670

23. Maijala R, Haukisalmi V, Henttonen $H$, Hirvelä-Koski V, Kauhala K, Kilpelä S-S, et al. Risk assessment on Echinococcus granulosus in Finland. Helsinki: National Veterinary and Food Research Institute;2002. 